04

\title{
Оценка временного ресурса работы катода электродугового плазмотрона постоянного тока
}

\author{
(C) В.М. Кулыгин, А.В. Переславцев, С.С. Тресвятский \\ Национальный исследовательский центр „Курчатовский институт“, \\ 123182 Москва, Россия \\ e-mail: Kulygin_VM@nrcki.ru
}

(Поступило в Редакцию 31 августа 2015 г. В окончательной редакции 8 декабря 2016 г.)

\begin{abstract}
Временной ресурс работы катода электродугового плазмотрона постоянного тока непрерывного действия с трубчатыми электродами, работающего на воздухе, рассмотрен с использованием полуфеноменологического подхода. В качестве основных физических явлений, составляющих рабочий процесс, приняты: термоэмиссия, обеспечивающая необходимый поток электронов и испарение материала катода, определяющее его эррозию. Используя известные закономерности этих явлений и имеющиеся в литературе экспериментальные данные, получены рабочие соотношения, позволяющие оценить время работы катода. Полученные при этом оценки удовлетворительно совпадают с результатами ресурсных испытаний.
\end{abstract}

DOI: 10.21883/JTF.2017.09.44904.1572

\section{Введение}

Временной ресурс работы электродуговых плазмотронов постоянного тока, предназначенных для реализации непрерывных технологических процессов, является одним из важных показателей их надежности и экономичности самого процесса. Как правило, он определяется ресурсом работы катодного узла плазмотрона, который, в свою очередь, связан с деградацией его термоэмиссионных элементов.

В настоящей работе нас будет интересовать электродуговой плазмотрон постоянного тока непрерывного действия с трубчатыми электродами (ЭДП). Типичная схема такого устройства приведена на рис. 1. Она включает катод $(A)$ с одной или несколькими термоэмиссионными вставками $(B)$ из тугоплавкого материала (при работе с кислородосодержащими газовыми смесями обычно используется гафний), устройство подачи рабочего газа $(C)$, с тангенциальной закруткой газового потока, выполненное из электроизолирующего материала, анод $(D)$, на торцевую поверхность которого $(I)$ выносится бо́льшая часть разрядного тока.

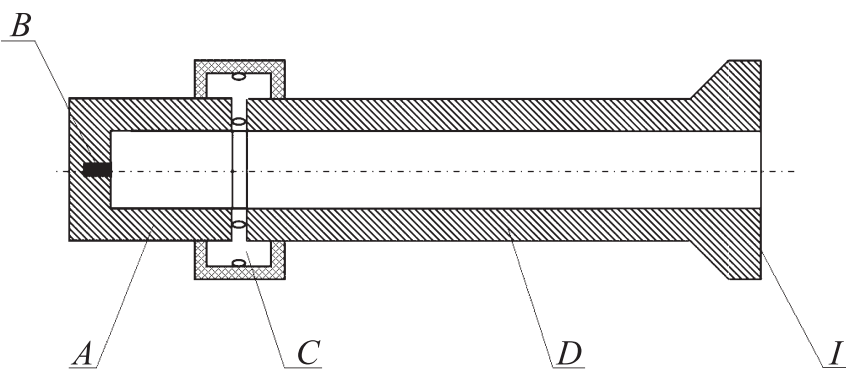

Рис. 1. Схема плазмотрона (пояснения в тексте).

\section{Процессы, учитываемые в рассматриваемой модели}

Следуя методу авторов работы [1], будем использовать для оценок известные закономерности процессов, выбранных в качестве рабочей модели, и сравнивать результаты этих оценок с доступными экспериментальными данными. Будем считать, что временной ресурс катода плазмотрона определяется скоростью испарения термоэмиссионных вставок, обеспечивающих необходимую эмиссию электронов. Плотность тока эмиссии, обеспечиваемая вставкой из гафния или циркония, вычисляем по формуле, представляющей собой закон Ричарсона-Дэшмана, скорректированный с учетом эффекта Шоттки, увеличивающим эмиссию за счет прикатодного падения потенциала [1]:

$$
j_{e k}=A T^{2} \exp \left(-\frac{e \varphi-e^{1.5} \sqrt{E /\left(4 \pi \varepsilon_{0}\right)}}{k T}\right),
$$

где $A=120.4 \mathrm{~A} \mathrm{~cm}^{-2} \mathrm{~K}^{-2}, e \varphi-$ работа выхода электрона (для гафния $3.53 \mathrm{eV}$, для циркония $3.9 \mathrm{eV}$ ), $E-$ напряженность электрического поля вблизи поверхности эмиссии (оцениваемое величиной $10^{8} \mathrm{~V} / \mathrm{m}[1]$ ).

Считая вслед за авторами [1], что требующийся для работы устройства ток эмиссии составляет $80-90 \%$ от тока разряда, примем для гафниевой вставки диаметром $2 \mathrm{~mm}$ необходимость обеспечивать не менее $160 \mathrm{~A}$ электронного тока. Если даже (в пределе) однородно эмитирует вся поверхность (площадь в $0.0314 \mathrm{~cm}^{2}$ ), требующаяся плотность тока составит $5096 \mathrm{~A} / \mathrm{cm}^{2}$. Оценивая температуру, при которой гафниевая вставка может выдать такую плотность тока, получаем величину $\sim 3000 \mathrm{~K}$. Отсюда видно, что нужная эмиссия обеспечивается при наличии на поверхности вставки слоя жидкого гафния (температура его плавления 2493 К). 


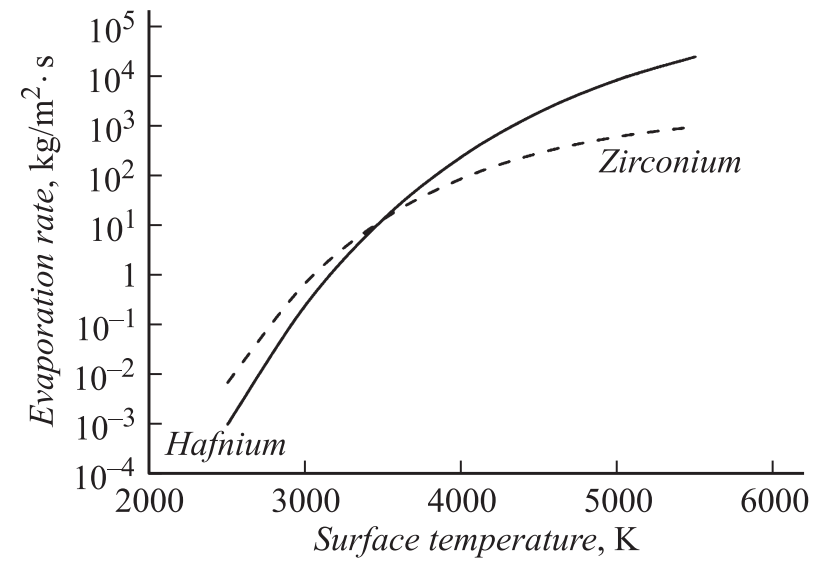

Рис. 2. Скорость испарения гафния и циркония в вакуум в зависимости от температуры поверхности.

Скорость испарения жидкости с единицы поверхности описывается уравнением Ленгмюра-Кнудсена

$$
\frac{d m}{d t}=k p \sqrt{\frac{M}{2 \pi R T}},
$$

где $T$ - температура поверхности испарения, $\mathrm{K} ; M-$ молекулярная масса жидкости $\left(M_{\mathrm{Hf}}=178.5, M_{\mathrm{Zr}}=91\right)$, $R=8.314 \mathrm{~J} / \mathrm{mol} \mathrm{K} ; p$ - равновесное давление пара над поверхностью, Па; $k$ - поправочный коэффициент, связанный с условиями вблизи поверхности испарения и на ней. Значение $k=1$ соответствует испарению с чистой поверхности в вакуум. Наличие рабочего газа, давление которого препятствует удалению пара от поверхности, уменьшает этот коэффициент, однако движение газа, „сдувающее“ и уносящее пар от испаряющей поверхности, его увеличивает. Кроме того, при использовании воздуха в качестве рабочего плазмообразующего газа должно оказывать влияние образование пленки оксида/нитрида на поверхности расплава, затрудняющей испарение. Не вдаваясь в детализацию степени влияния всех этих трудно учитываемых количественно факторов, будем рассматривать коэффициент $k$ как параметр, значение которого следует определять из сравнения получаемых оценок с доступными экспериментальными данными.

В интересующем нас диапазоне температур зависимость равновесного давления паров металла вставки над поверхностью от ее температуры, взятая из справочника [2], с хорошей точностью может быть аппроксимирована выражением

$$
p_{\text {vap }}=\frac{10^{\Lambda}}{7.5 \cdot 10^{-3}} \mathrm{~Pa}
$$

где $\Lambda=5.13-44.5 \exp (-T k / 1548)$ для гафния, $\Lambda=$ $=3-52.3 \exp (-T k / 1130)$ для циркония. Скорости испарения гафния и циркония в зависимости от температуры испаряющей поверхности, сосчитанная по формуле (2) при $k=1$, приведена на рис. 2.
Таким образом, интенсивность двух интересующих нас процессов: эмиссии электронов, необходимой для горения дугового разряда в плазмотроне, и испарения материала катода, ограничивающего временной ресурс его работы, зависят от температуры поверхности.

\section{Рассматриваемые результаты экспериментов и их обсуждение}

Фундаментальным показателем, отражающим ресурсные возможности материала катода, является удельное испарение - отношение скорости испарения материала катода с единицы поверхности к плотности эмитируемого тока, выражающееся в количестве испарившегося вещества на кулон эмитируемых катодом электронов. Естественно, подразумевается примерное равенство эмитируемой и испаряющей поверхностей.

В реальных условиях работы плазмотрона измеряемой величиной является обычно ток разряда, на 80-90\% состоящий из тока эмиссии электронов с катода, дополняемого током приходящих на него ионов. В связи с этим в условиях эксплуатации берут в качестве базы для определения удельного испарения именно ток разряда.

На рис. 3 представлен набор данных по удельной эрозии катодов, приведенный в работе [3] и представляющий собой диаграмму, на которой размещены значения удельной эрозии, полученные при различных значениях разрядного тока. Хотя, если испарение материала принимается в качестве механизма эрозии катода, важна температура его поверхности, а ток разряда при одинаковой температуре будет разным для циркония и гафния (разная работа выхода), эти данные можно использовать для ориентировочной оценки. Сплошными линиями мы ограничили сверху области расположения экспериментальных точек для результатов с гафниевыми (нижняя линия) и циркониевыми (верхняя линия) вставками. Оставляя за рамками наших рассуждений причины впечатляющего разброса экспериментальных точек, отражающего, по-видимому, различные условия экспериментов, обратим внимание на то, что значения

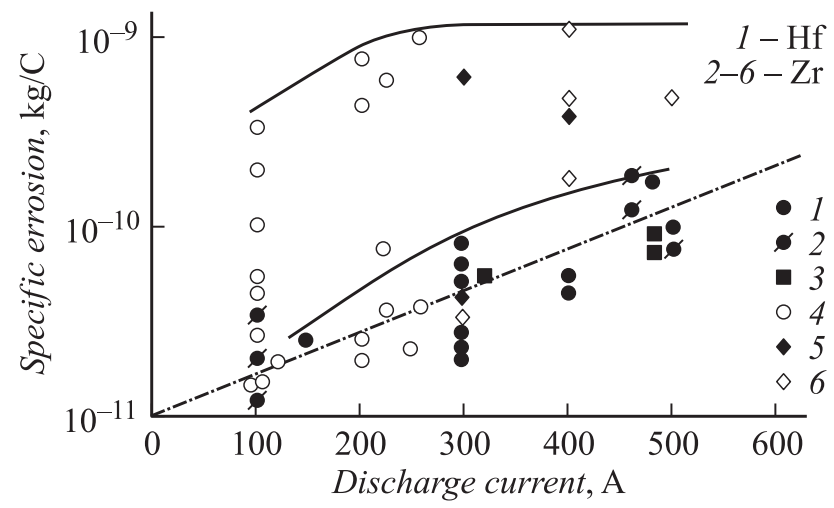

Рис. 3. Статистика результатов экспериментов с гафниевыми и циркониевыми вставками из работы [3]. 


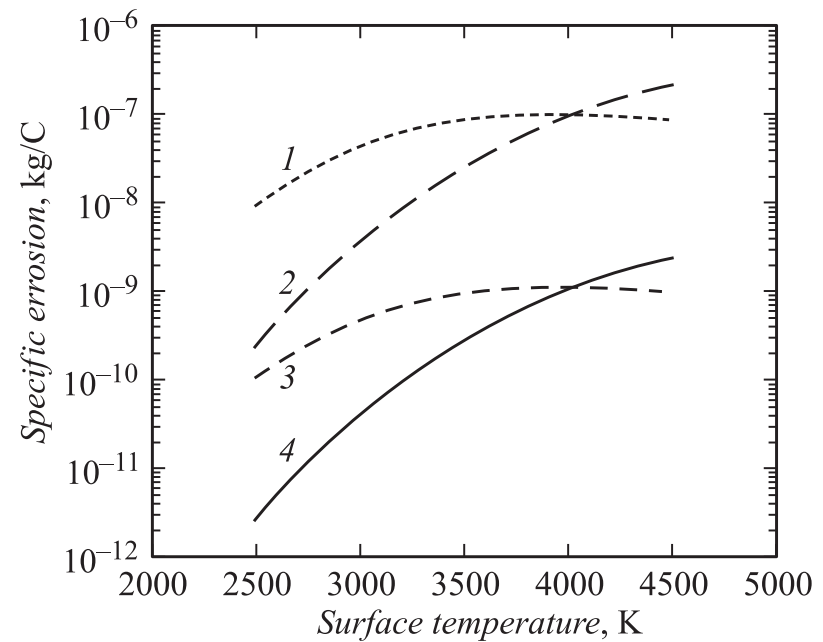

Рис. 4. Удельное испарение материала катода в зависимости от температуры его поверхности: 1 - цирконий, 2 - гафний при испарении в вакуум $(k=1), 3$ - цирконий и 4 - гафний в рабочих условиях $(k=0.011)$.

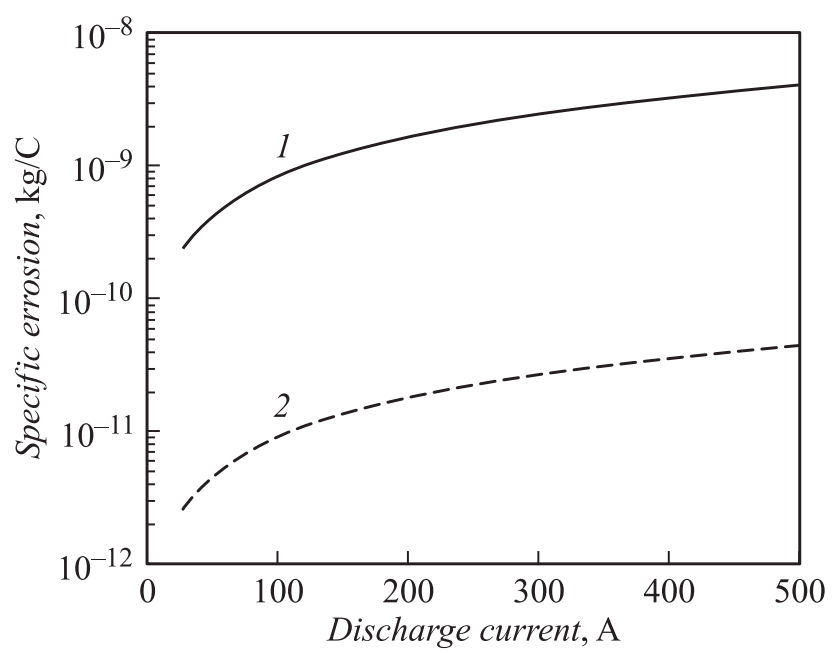

Рис. 5. Удельное испарение материала катода в зависимости от тока разряда на гафниевую вставку диаметром $3 \mathrm{~mm}: 1-$ расчет при испарении в вакуум $(k=1), 2$ - корректировка в соответствии со статистикой [3] $(k=0.011)$. Ток разряда принимался равным току эмиссии + ионному току $(15 \%)$.

удельного испарения для циркония не выходят за уровень $\sim 10^{-9} \mathrm{~kg} / \mathrm{C}$, в то время, как на рис. 4 кривая 1 , построенная для циркония, испаряющегося в вакуум, доходит до величин $\sim 10^{-7} \mathrm{~kg} / \mathrm{C}$. Примерно такое же отличие кривой 2 (гафний) от нижней линии рис. 3 . Это можно истолковать как понижение примерно на два порядка величины реальной скорости испарения материала катода плазмотрона при рабочих условиях по сравнению с испарением в вакуум. Кривые 3 и 4 рис. 4 построены для циркония и гафния соответственно с введением корректирующего коэффициента $k=0.011$ в формуле (2) .
Для более аккуратного сопоставления с ограничениями, представленными на рис. 3, следует использовать связь тока разряда с температурой катодной поверхности, учитывая реальную площадь поверхности эмиссии. На рис. 5 представлены результаты такого расчета для гафниевой вставки диаметром $3 \mathrm{~mm}$.

\section{Ресурсные испытания катода плазмотрона ЭДП-200}

Используем теперь полученную „калибровку“ для трактовки результатов ресурсных испытаний катода плазмотрона ЭДП-200 [4]. Катод представляет собой медный „стакан“, в дно которого запрессованы семь гафниевых вставок диаметром $3 \mathrm{~mm}$ и длиной $10 \mathrm{~mm}$, одна центральная и шесть периферийных (рис. 6).

В ходе проведенных испытаний ток дуги поддерживался на уровне $130 \mathrm{~A} \mathrm{в} \mathrm{течение} 350 \mathrm{~h}$. Испытания подтвердили, что в принятой конфигурации катода дуга „садится“ на гафниевые вставки, причем наибольшей эрозии подвергается центральная вставка. Ее выработка за время испытаний составила $\sim 2 \mathrm{~mm}$. В то время как выработка периферийных вставок оказалась на уровне 0.8-1.2 mm. Существенных изменений режима горения дуги в ходе испытаний не наблюдалось.

Приняв среднее укорочение периферийных вставок на $1 \mathrm{~mm}$ (полное $\Delta L=2+1 \cdot 6=8 \mathrm{~mm}$ ), получаем суммарное уменьшение массы гафния в 3-х миллиметровых вставках

$$
\Delta M=\pi \frac{d^{2}}{4} \Delta L \rho_{\mathrm{Hf}}=3.14 \frac{0.3^{2}}{2} \cdot 0.8 \cdot 12.5=0.706 \mathrm{~g}
$$

(плотность гафния $\rho_{\mathrm{Hf}}=12.5 \mathrm{~g} / \mathrm{cm}^{3}$ ).

Указанная потеря массы произошла за время $\Delta t=$ $=350 \cdot 3600=1,26 \cdot 10^{6} \mathrm{~s}$. Таким образом, используемое обычно в качестве показателя, характеризующего износ катодных вставок, отношение скорости потери

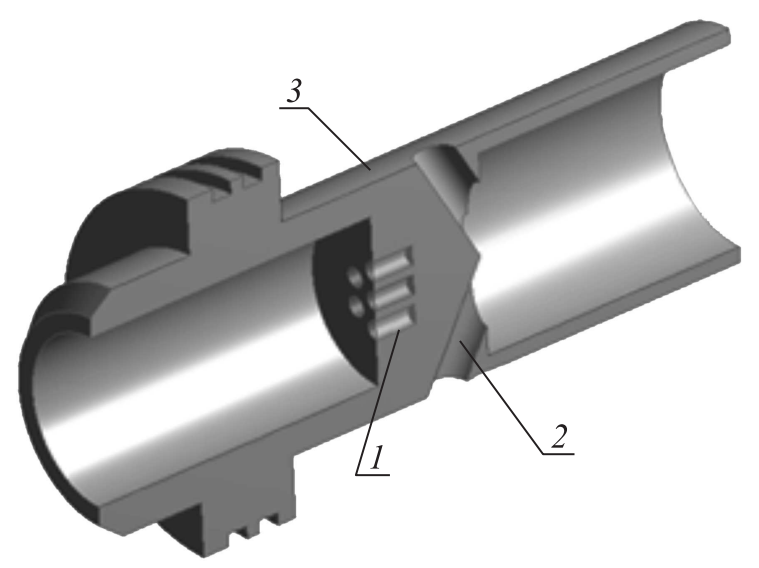

Рис. 6. Катод плазмотрона ЭДП-200. Внешний вид с разрезом: 1 - гафниевые вставки, 2 - каналы охлаждения, 3 „рубашка“ охлаждения катода. 


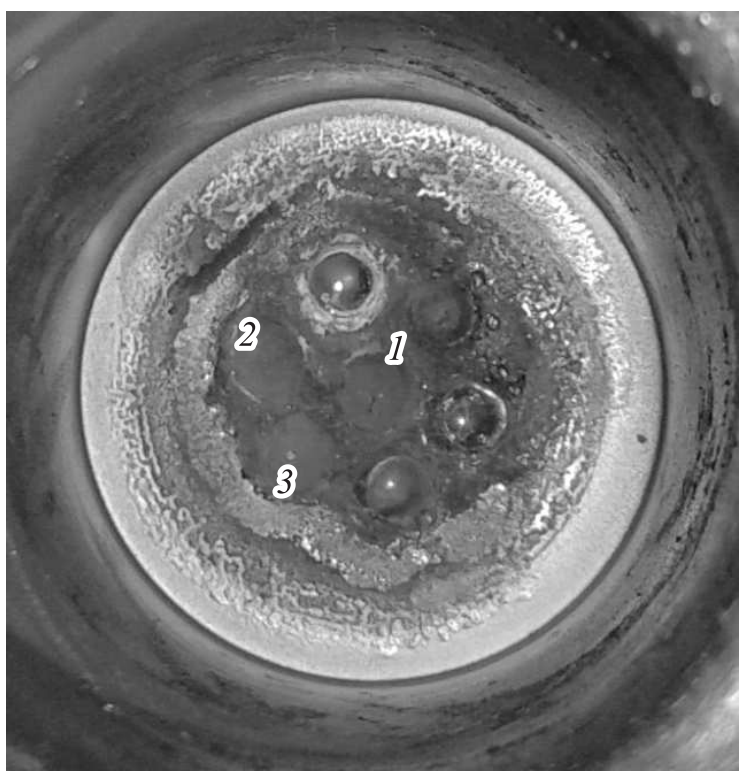

Рис. 7. Состояние торцевой части (дна) катода после $350 \mathrm{~h}$ работы.

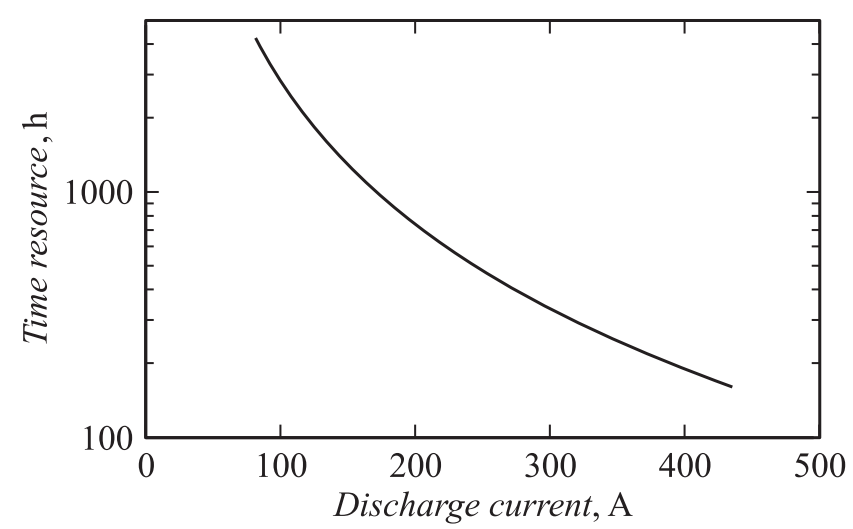

Рис. 8. Зависимость ресурса работы катода плазмотрона ЭДП-200 от тока разряда при $\Delta M_{\mathrm{cr}}=3.3 \cdot 10^{-3} \mathrm{~kg}$.

массы к величине разрядного тока составило в этом случае

$$
G_{\mathrm{ex}}=\frac{\Delta M}{\Delta t \cdot 130 \cdot 10^{3}} \approx 4 \cdot 10^{-12} \mathrm{~kg} / \mathrm{C}
$$

Поскольку отличие величины тока разряда от его эмиссионной составляющей в рабочем диапазоне испытываемого плазмотрона обычно не превышает 10-20\%, можем использовать данные, приведенные на рис. 5 (кривая 2), для определения с оговоренной точностью эффективной температуры работы катодных вставок. Видно, что удельной потере массы в $4 \cdot 10^{-12} \mathrm{~kg} / \mathrm{C}$ соответствует эффективная температура поверхности $\approx 2600 \mathrm{~K}$.

С помощью формулы (1) находим, что температуре $2600 \mathrm{~K}$ для вставки диаметром $0.3 \mathrm{~cm}$ соответствует ток

\begin{tabular}{l|c|c|c|c}
\hline \multicolumn{1}{c|}{ Диаметр вставки, ст } & 0.16 & 0.2 & 0.25 & 0.30 \\
\hline $\begin{array}{l}\text { Предельный ток разряда, A } \\
\text { Прикатодное напряжение }\end{array}$ & 80 & 200 & 300 & 400 \\
\hline $\begin{array}{l}\text { at } K f=10 \\
\text { at } K f=5\end{array}$ & 23.566 & $\begin{array}{c}11.783 \\
5.891\end{array}$ & $\begin{array}{l}9.819 \\
4.91\end{array}$ & $\begin{array}{l}8.837 \\
4.419\end{array}$
\end{tabular}

эмиссии $\approx 45 \mathrm{~A}$. Разделив рабочий ток разряда $(130 \mathrm{~A})$ на эту величину, получаем

$$
130 / 45=2.889 \text {. }
$$

То есть эффективно работали примерно 3 вставки из 7, что и подтверждается фотографией на рис. 7.

Видно, что в наибольшей степени выработаны три вставки из семи: центральная и две периферийных (с левой стороны рисунка).

Итак, если ресурсом работы катода считать время испарения некоторой критической массы $\left(\Delta M_{\mathrm{cr}}, \mathrm{kg}\right)$ материала вставки, то этот ресурс может быть рассчитан по формуле:

$$
\tau(\mathrm{h})=\frac{\Delta M_{\mathrm{cr}}}{G \cdot I_{p} \cdot 3600} .
$$

При расчете зависимости, представленной на рис. 8, ток разряда рассчитывался по формуле

$$
I_{p}=j_{e k} \cdot 1.15 \cdot S_{\mathrm{eff}}
$$

где учтен вклад ионного тока $(15 \%)$, а $S_{\text {eff }}=3 \cdot S_{\text {insert }}-$ учет числа активно работавших вставок. Величина $G$ рассчитывалась в соответствии с условиями, принятыми при расчетах ее зависимости от температуры, результаты которых представлены на рис. 5.

Вернемся теперь к возможным причинам резкого уменьшения испарения материала катодной вставки по сравнению с испарением в вакуум.

В работе [3] установлено существование предела по разрядному току, превышение которого приводит к быстрому разрушению катодной вставки. В таблице (в первой и второй строках) приведены критические значения тока для вставок различного диаметра, взятые из [3].

Сама „пороговость“ явления наводит на мысль о фазовом переходе, лежащим в его основе. Предположив, что испарение материала вставки ограничено наличием оксидно/нитридной корки, разрушаемой при достижении температуры ее плавления, проведем грубую размерностную оценку правдоподобности этого механизма.

Считаем, что мощность, выделяющаяся на вставку $P \sim I \cdot U$, отводится в основном за счет теплопроводности, растекаясь внутри вставки. Поток тепла $Q=K f d^{2} \chi \Delta T / d$, где $d$ - диаметр вставки, $\chi-$ коэффициент теплопроводности гафния $(36 \mathrm{~W} / \mathrm{mK}), \Delta T-$ разность температур между вставкой и медной частью катода, $K f$ - безразмерный коэффициент, учитывающий форму растекания тепла и неточность выражения 
для мощности, идущей на нагрев, что позволяет нам записать

$$
U I=K f d^{2} \chi \Delta T / d .
$$

Отсюда следует связь между значением критического тока и диаметром вставки

$$
I_{\mathrm{cr}}=\frac{K f \chi \Delta T d}{U} .
$$

Принимая $\Delta T=T_{\mathrm{cr}}-T_{0}=3573-300 \mathrm{~K} \quad(3573 \mathrm{~K}-$ температура плавления нитрида гафния, оксид плавится при $T=3053-3063 \mathrm{~K})$ и $K f=10$ и 5, получаем результаты, соответствующие приведенным экспериментальным значениям $I_{\mathrm{cr}}$ при значениях прикатодного падения $U$, приведенных в третьей строке таблицы. Эти значения соответствуют по порядку величины оценкам прикатодного падения в работе [4].

\section{Обсуждение и выводы}

Использование полуфеноменологического подхода, заключающегося в построении упрощенной качественной модели сложного, трудно поддающегося прямому теоретическому описанию процесса с последующей ее „подгонкой“ под имеющиеся экспериментальные результаты, дает возможность прояснить представления о его природе и служит основанием для разработки расчетных моделей. Такой подход особенно полезен, когда практически невозможны прямые измерения локальных параметров, характеризующих процесс.

Действительно, приняв за основу два фундаментальных явления, характеризующих материалы, используемые для изготовления термоэмиссионных элементов катодных узлов плазмотронов, и, сравнивая результаты расчетов с результатами интегральных измерений, мы получили

- уточнение характеристики „удельное испарение“ $(G, \mathrm{~kg} / \mathrm{C})$ как отношения скорости испарения к плотности тока эмиссии. При ее определении в условиях вакуума $\left(G_{0}\right)$ она становится фундаментальной характеристикой материала катода,

- количественную оценку степени уменьшения $G$ (за счет скорости испарения материала катода) в условиях работы плазмотрона по сравнению со скоростью испарения с чистой поверхности в вакуум,

- схему расчета ресурса работы катода плазмотрона,

- косвенное подтверждение предположения о механизме эффекта, ответственного за наличие предельного тока разряда и зависимости его величины от диаметра вставки,

- приведенные результаты позволяют сделать вывод о том, что использованный подход может быть полезен при количественных оценках ресурса работы катодных узлов в плазмотронах рассмотренного типа,

- при этих оценках следует иметь в виду, что процесс считается стационарным, все параметры представлены средними (эффективными) значениями, диапазон температур

$$
T_{\text {melt }}<T<T_{\text {boil }}
$$

\section{Список литературы}

[1] Гнеденко В.Г., Иванов А.А., Переславцев А.В., Тресвятский C.C. // Вопросы атомной науки и техники. Сер. Плазменная электроника и новые методы ускорения. 2006. № 5. C. $75-79$.

[2] Таблицы физических величин / Под ред. И.К. Кикоина. М.: Атомиздат, $1976.1005 \mathrm{c}$.

[3] Аньшаков А.С., Бутова М.Н. и др. // Тр. V Всесоюз. конф. по генераторам низкотемпературной плазмы. Новосибирск: Институт теплофизики СО АН СССР, 1972. Т. 2. С. 48-52.

[4] Gnedenko V.G., Ivanov A.A., Komarov N.S., Mondgeran Ju.P., Pereslavtsev A.V., Titov V.V., Tresviatsky S.S. // V Int. Conf. Plasma Phys. Plasma Technol. Minsk, Belarus, 2006. P. 715-718. 